\title{
Normal forms for singularities of pedal curves produced by non-singular dual curve germs in $S^{n}$
}

\author{
Takashi Nishimura
}

Received: date / Accepted: date

\begin{abstract}
For an $n$-dimensional spherical unit speed curve $\mathbf{r}$ and a given point $P$, we can define naturally the pedal curve of $\mathbf{r}$ relative to the pedal point $P$. When the dual curve germs are non-singular, singularity types of such pedal curves depend only on locations of pedal points. In this paper, we give a complete list of normal forms for singularities and locations of pedal points when the dual curve germs are non-singular. As an application of our list, we characterize $C^{\infty}$ left equivalence classes of pedal curve germs $\left(I, s_{0}\right) \rightarrow S^{n}$ produced by non-singular dual curve germ from the viewpoint of the relation between $\mathcal{L}$ tangent space and $\mathcal{C}$ tangent space.
\end{abstract}

Keywords normal form $\cdot$ singularity $\cdot$ pedal curve $\cdot$ pedal point $\cdot$ dual curve $\cdot$ map of blow up type

Mathematics Subject Classification (2000) 57R45 -58C25 -53A40 - 53A04

\section{Introduction}

Let $I$ be an open interval and $S^{n}$ be the $n$-dimensional unit sphere in $\mathbf{R}^{n+1}$. A $C^{\infty}$ regular map $\mathbf{r}: I \rightarrow S^{n}$ is said to be a spherical unit speed curve if each of the following $\mathbf{u}_{i}(s) \quad(1 \leq i \leq n-1)$ is inductively well-defined for any $s \in I$ (in other words, each of the following $\kappa_{i}(s) \quad(1 \leq i \leq n-1)$ is a positive function), where initial information are $\mathbf{u}_{-1}(s) \equiv \mathbf{0}, \mathbf{u}_{0}(s)=\mathbf{r}(s),\left\|\mathbf{u}_{0}^{\prime}(s)\right\| \equiv 1$ and $\kappa_{0}(s) \equiv 0$.

$$
\begin{array}{ll}
\mathbf{u}_{i}(s)=\frac{\mathbf{u}_{i-1}^{\prime}(s)+\kappa_{i-1}(s) \mathbf{u}_{i-2}(s)}{\left\|\mathbf{u}_{i-1}^{\prime}(s)+\kappa_{i-1}(s) \mathbf{u}_{i-2}(s)\right\|} & (1 \leq i \leq n-1) \\
\kappa_{i}(s)=\left\|\mathbf{u}_{i-1}^{\prime}(s)+\kappa_{i-1}(s) \mathbf{u}_{i-2}(s)\right\| & (1 \leq i \leq n-1)
\end{array}
$$

Note that the above inductive conditions for a spherical unit speed curve $\mathbf{r}$ are not so strong restrictions. This is because first by using Thom transversality theorem (for

Takashi Nishimura

Department of Mathematics, Faculty of Education and Human Sciences, Yokohama National University, Yokohama240-8501, Japan

E-mail: takashi@edhs.ynu.ac.jp 
instance, see [6]) $(n-2)$ times for any $C^{\infty}$ regular map $\mathbf{r}: I \rightarrow S^{n}$ we can obtain a sufficiently near $C^{\infty}$ map $\widetilde{r}$ in $C^{\infty}\left(I, S^{n}\right)$ with Whitney $C^{\infty}$ topology such that

$$
\widetilde{\mathbf{r}}(s), \frac{d \widetilde{\mathbf{r}}}{d s}(s), \cdots, \frac{d^{n-1} \widetilde{\mathbf{r}}}{d s^{n-1}}(s) \text { are linearly independent for any } s \in I .
$$

Then the so-called arc-length parameter gives a $C^{\infty}$ diffeomorphism $h: I \rightarrow I$ such that $\widetilde{\mathbf{r}} \circ h^{-1}$ is a spherical unit speed curve.

For a spherical unit speed curve we see that any two of $\mathbf{u}_{i}, \mathbf{u}_{j} \quad(0 \leq i, j \leq$ $n-1, i \neq j$ ) are perpendicular (see $\S 2$ ). Therefore, we can define one more vector $\mathbf{u}_{n}(s)$ uniquely so that $\left\{\mathbf{u}_{0}(s), \mathbf{u}_{1}(s), \cdots, \mathbf{u}_{n}(s)\right\}$ is an orthogonal moving frame and $\operatorname{det}\left(\mathbf{u}_{0}(s), \cdots, \mathbf{u}_{n}(s)\right)=1$ for any $s \in I$. The map $\mathbf{u}_{n}: I \rightarrow S^{n}$ is called the dual curve of $\mathbf{r}([1])$. By using the dual curve $\mathbf{u}_{n}$ we define $\kappa_{n}$ as follows, where the dot in the center means the scalar product:

$$
\kappa_{n}(s)=\mathbf{u}_{n-1}^{\prime}(s) \cdot \mathbf{u}_{n}(s) .
$$

We see that the dual curve $\mathbf{u}_{n}$ is non-singular at $\mathrm{s}$ if and only if $\kappa_{n}(s) \neq 0$ (see $\S 2$ ).

For any $i \quad(-1 \leq i \leq n)$, we put

$$
S_{\mathbf{u}_{i}(s)}^{i}=\left(S^{n}-\left\{ \pm \mathbf{u}_{n}(s)\right\}\right) \cap \sum_{j=-1}^{i} \mathbf{R u}_{j}(s) .
$$

Given a spherical unit speed curve $\mathbf{r}: I \rightarrow S^{n}$, choosing a point $P$ of $S^{n}-\left\{ \pm \mathbf{u}_{n}(s) \mid s \in\right.$ $I\}$ gives the map which maps $s \in I$ to the unique nearest point in $S_{\mathbf{u}_{n-1}(s)}^{n-1}$ from $P$. Such a map is called the pedal curve relative to the pedal point $P$ for an $n$-dimensional unit speed curve $\mathbf{r}$ and is denoted by $p e d_{\mathbf{r}, P}$. Note that since all points in $S_{\mathbf{u}_{n-1}(s)}^{n-1}$ are the nearest points from $\pm \mathbf{u}_{n}(s)$ the pedal point $P$ for the map-germ $\operatorname{ped}_{\mathbf{r}, P}$ at $s$ must be outside $\left\{ \pm \mathbf{u}_{n}(s)\right\}$.

The purpose of this paper is to show the following.

Theorem 1.1 Let $\mathbf{r}$ be an $n$-dimensional spherical unit speed curve. Let $s_{0} \in I$ be such that $\kappa_{n}\left(s_{0}\right) \neq 0$. Then the following hold.

1. The pedal point $P$ is inside $S_{\mathbf{u}_{n}\left(s_{0}\right)}^{n}-S_{\mathbf{u}_{n-2}\left(s_{0}\right)}^{n-2}$ if and only if the map-germ ped $\mathbf{r}_{\mathbf{r}, P}$ : $\left(I, s_{0}\right) \rightarrow S^{n}$ is $C^{\infty}$ left equivalent to the map-germ given by $s \mapsto(s, 0, \cdots, 0)$.

2. For any $i(0 \leq i \leq n-2)$, the pedal point $P$ is inside $S_{\mathbf{u}_{i}\left(s_{0}\right)}^{i}-S_{\mathbf{u}_{i-1}\left(s_{0}\right)}^{i-1}$ if and only if the map-germ ped $d_{\mathbf{r}, P}:\left(I, s_{0}\right) \rightarrow S^{n}$ is $C^{\infty}$ left equivalent to the map-germ given by the following:

$$
s \mapsto(\underbrace{s^{n-i}, s^{n-i+1}, \cdots, s^{2 n-2 i-1}}_{(n-i) \text { elements }}, \underbrace{0, \cdots, 0}_{i \text { elements }}) .
$$

Here, two map-germs $f, g:(\mathbf{R}, 0) \rightarrow\left(\mathbf{R}^{n}, 0\right)$ are said to be $C^{\infty}$ left equivalent if there exist a germ of $C^{\infty}$ diffeomorphism $h_{t}:\left(\mathbf{R}^{n}, 0\right) \rightarrow\left(\mathbf{R}^{n}, 0\right)$ such that the identity $g=h_{t} \circ f$ is satisfied.

As a corollary of theorem 1.1, we can characterize $C^{\infty}$ left equivalence classes of pedal curve germs $\left(I, s_{0}\right) \rightarrow S^{n}$ with $\kappa_{n}\left(s_{0}\right) \neq 0$ from the viewpoint of the relation between $\mathcal{L}$ tangent space and $\mathcal{C}$ tangent space. For the definitions of $\mathcal{L}$ tangent space and $\mathcal{C}$ tangent space, see [7] or [9]. Let $\mathcal{O}(1, n)$ be the set of $C^{\infty}$ map-germs $f:(\mathbf{R}, 0) \rightarrow$ 
$\left(\mathbf{R}^{n}, 0\right)$ such that $T \mathcal{L}(f)=T \mathcal{C}(f)(\mathcal{O}$ means "open") with finite codimensions ; and let $\mathcal{P}(1, n)$ be the set of $C^{\infty}$ map-germs $(\mathbf{R}, 0) \rightarrow\left(\mathbf{R}^{n}, 0\right)$ which are $C^{\infty}$ left equivalent to some pedal curve germ $\left(I, s_{0}\right) \rightarrow S^{n}$ with $\kappa_{n}\left(s_{0}\right) \neq 0$ ( $\mathcal{P}$ means "pedal"). Since any normal form in theorem 1.1 belongs to $\mathcal{O}(1, n)$, any map-germ in $\mathcal{P}(1, n)$ is $C^{\infty}$ left equivalent to one of normal forms in theorem 1.1 and any map-germ in $\mathcal{O}(1, n)$ is $C^{\infty}$ left equivalent to one of normal forms in theorem $1.1^{1}$, we have the following.

Corollary $1.1 \mathcal{O}(1, n)=\mathcal{P}(1, n)$

Note that it is impossible to obtain the same result as in corollary 1.1 if we replace $T \mathcal{L}(f)=T \mathcal{C}(f)$ with $T \mathcal{A}(f)=T \mathcal{K}(f)$ in the definition of $\mathcal{O}(1, n)$ since the equality $T \mathcal{A}(f)=T \mathcal{K}(f)$ holds even for $f(s)=\left(s^{3}, s^{4}\right)$. Thus, in our situation the $C^{\infty}$ right-left equivalence does not work well, but the $C^{\infty}$ left equivalence does so. This is a merit since the $C^{\infty}$ left equivalence is easy to deal with as pointed out in [4]. Furthermore, in our situation we can truncate higher terms quite easily by using Malgrange preparation theorem only one time, we need no calculations by using semigroups as in [2] (see §5). On the other hand, note also that it is impossible in general to characterize $P(1, n)$ as the set of tops of hierarchies of $\mathcal{A}$-simple singularities since normal forms in 2 of theorem 1.1 are $\mathcal{A}$-simple if and only if $n \leq 6$ due to [2]. Thus, it seems that the notion of simple singularity is not suitable for singularities of pedal in general.

In $\S 2$ we investigate several properties of the set $\left\{\mathbf{u}_{0}(s), \cdots, \mathbf{u}_{n}(s)\right\}$. $\S 3$ is devoted to factor $\operatorname{ped}_{\mathbf{r}, P}$ as the composition of the dual curve, the canonical projection and one map $\widetilde{\Psi}_{P}$. In $\S 4$ we show that the map $\widetilde{\Psi}_{P}$ introduced in $\S 3$ is $C^{\infty}$ right-left equivalent to the blow up of $\mathbf{R}^{n}$ at the origin. Proof of theorem 1.1 is given in $\S 5$.

\section{Several properties of the set $\left\{\mathbf{u}_{0}(s), \cdots, \mathbf{u}_{n}(s)\right\}$}

Lemma 2.1 For any $s \in I$ and any $i, k \quad(-1 \leq i<k \leq n-1)$ the following three hold.

$$
\begin{aligned}
\mathbf{u}_{i}(s) \cdot \mathbf{u}_{k}(s) & =0 \\
\mathbf{u}_{i}(s) \cdot \mathbf{u}_{k}^{\prime}(s) & =0 \quad(i<k-1) \\
\mathbf{u}_{k-1}(s) \cdot \mathbf{u}_{k}^{\prime}(s) & =-\kappa_{k}(s)
\end{aligned}
$$

Proof of lemma 2.1 We show lemma 2.1 by induction on $k$.

First, by definitions it is trivial that $\mathbf{u}_{-1}(s) \cdot \mathbf{u}_{0}(s)=0$ and $\mathbf{u}_{-1}(s) \cdot \mathbf{u}_{0}^{\prime}(s)=-\kappa_{0}(s)$. Next, we assume that for any $i, j \quad(-1 \leq i<j<k \leq n-1)$, the following three hold.

$$
\begin{aligned}
\mathbf{u}_{i}(s) \cdot \mathbf{u}_{j}(s) & =0 \\
\mathbf{u}_{i}(s) \cdot \mathbf{u}_{j}^{\prime}(s) & =0 \quad(i<j-1) \\
\mathbf{u}_{j-1}(s) \cdot \mathbf{u}_{j}^{\prime}(s) & =-\kappa_{j}(s)
\end{aligned}
$$

\footnotetext{
1 The last assertion on map-germs in $\mathcal{O}(1, n)$ is easily obtained by Gaffney's criterion on $\mathcal{L}$-equivalence (for Gaffney's criterion on $\mathcal{L}$-equivalence, see theorem 2.7 of [9]).
} 
Under this assumption, we see that

$$
\begin{aligned}
\mathbf{u}_{k-2}(s) \cdot \mathbf{u}_{k}(s) & =\frac{1}{\kappa_{k}(s)} \mathbf{u}_{k-2}(s) \cdot\left(\mathbf{u}_{k-1}^{\prime}(s)+\kappa_{k-1}(s) \mathbf{u}_{k-2}(s)\right) \\
& =\frac{1}{\kappa_{k}(s)}\left(-\kappa_{k-1}(s)+\kappa_{k-1}(s)\right)=0
\end{aligned}
$$

and for $i<k, i \neq k-2$ we see that

$$
\begin{aligned}
\mathbf{u}_{i}(s) \cdot \mathbf{u}_{k}(s) & =\frac{1}{\kappa_{k}(s)} \mathbf{u}_{i}(s) \cdot\left(\mathbf{u}_{k-1}^{\prime}(s)+\kappa_{k-1}(s) \mathbf{u}_{k-2}(s)\right) \\
& =\frac{1}{\kappa_{k}(s)}(0+0)=0
\end{aligned}
$$

Next, under the same assumption we see that for any $i \quad(0 \leq i<k-1)$

$$
\begin{aligned}
\mathbf{u}_{i}(s) \cdot \mathbf{u}_{k}^{\prime}(s) & =-\mathbf{u}_{i}^{\prime}(s) \cdot \mathbf{u}_{k}(s) \\
& =-\left(\kappa_{i+1}(s) \mathbf{u}_{i+1}(s)-\kappa_{i}(s) \mathbf{u}_{i-1}(s)\right) \cdot \mathbf{u}_{k}(s) \\
& =-(0+0)=0
\end{aligned}
$$

and in the case that $i=k-1$ we see

$$
\begin{aligned}
\mathbf{u}_{k-1}(s) \cdot \mathbf{u}_{k}^{\prime}(s) & =-\mathbf{u}_{k-1}^{\prime}(s) \cdot \mathbf{u}_{k}(s) \\
& =-\left(\kappa_{k}(s) \mathbf{u}_{k}(s)-\kappa_{k-1}(s) \mathbf{u}_{k-2}(s)\right) \cdot \mathbf{u}_{k}(s) \\
& =-\left(\kappa_{k}(s)+0\right)=-\kappa_{k}(s) .
\end{aligned}
$$

Of course, $\mathbf{u}_{-1}(s) \cdot \mathbf{u}_{k}^{\prime}(s)=0$ holds under no assumption.

Therefore, lemma 2.1 is proved by induction.

Lemma 2.1 shows that $\left\{\mathbf{u}_{0}(s), \cdots, \mathbf{u}_{n}(s)\right\}$ is an orthogonal moving frame.

Lemma 2.2 For any $s \in I$ the following two hold.

1. $\mathbf{u}_{n-1}^{\prime}(s)=-\kappa_{n-1}(s) \mathbf{u}_{n-2}(s)+\kappa_{n}(s) \mathbf{u}_{n}(s)$,

2. $\mathbf{u}_{n}^{\prime}(s)=-\kappa_{n}(s) \mathbf{u}_{n-1}(s)$.

Proof of lemma 2.2 First we show 2 of lemma 2.2. By definition, for any $i \quad(i<$ $n-1)$

$$
\mathbf{u}_{i}^{\prime}(s) \cdot \mathbf{u}_{n}(s)=\left(\kappa_{i+1}(s) \mathbf{u}_{i+1}(s)-\kappa_{i}(s) \mathbf{u}_{i-1}(s)\right) \cdot \mathbf{u}_{n}(s)=0 .
$$

Thus, we have that $\mathbf{u}_{i}(s) \cdot \mathbf{u}_{n}^{\prime}(s)=0$. Combining this result with $\mathbf{u}_{n}^{\prime}(s) \cdot \mathbf{u}_{n}(s)=0$ implies that we may put $\mathbf{u}_{n}^{\prime}(s)=\alpha(s) \mathbf{u}_{n-1}(s)$. Then,

$$
\alpha(s)=\mathbf{u}_{n-1}(s) \cdot \mathbf{u}_{n}^{\prime}(s)=-\mathbf{u}_{n-1}^{\prime}(s) \cdot \mathbf{u}_{n}(s)=-\kappa_{n}(s) .
$$

Next, we show 1 of lemma 2.2. By similar arguments as in the proof of 2 of lemma 2.2 we may put $\mathbf{u}_{n-1}^{\prime}(s)=\beta(s) \mathbf{u}_{n-2}(s)+\kappa_{n}(s) \mathbf{u}_{n}(s)$. Then, lemma 2.1 and 2 of lemma 2.2 show that $\beta(s)=-\kappa_{n-1}(s)$. 
By lemma 2.2, we see that the dual curve $\mathbf{u}_{n}(s)$ is non-singular if and only if $\kappa_{n}(s) \neq 0$ and we obtain the following Serret Frenet type formula.

$$
\left(\begin{array}{c}
\mathbf{u}_{0}^{\prime}(s) \\
\mathbf{u}_{1}^{\prime}(s) \\
\mathbf{u}_{2}^{\prime}(s) \\
\vdots \\
\mathbf{u}_{n-2}^{\prime}(s) \\
\mathbf{u}_{n-1}^{\prime}(s) \\
\mathbf{u}_{n}^{\prime}(s)
\end{array}\right)=\left(\begin{array}{ccccccc}
0 & \kappa_{1}(s) & 0 & \cdots & 0 & 0 & 0 \\
-\kappa_{1}(s) & 0 & \kappa_{2}(s) & \ddots & 0 & 0 & 0 \\
0 & -\kappa_{2}(s) & 0 & \ddots & 0 & 0 & 0 \\
\vdots & \ddots & \ddots & \ddots & \ddots & \ddots & \vdots \\
0 & 0 & 0 & \ddots & 0 & \kappa_{n-1}(s) & 0 \\
0 & 0 & 0 & \ddots & -\kappa_{n-1}(s) & 0 & \kappa_{n}(s) \\
0 & 0 & 0 & \ddots & 0 & -\kappa_{n}(s) & 0
\end{array}\right)\left(\begin{array}{c}
\mathbf{u}_{0}(s) \\
\mathbf{u}_{1}(s) \\
\mathbf{u}_{2}(s) \\
\vdots \\
\mathbf{u}_{n-2}(s) \\
\mathbf{u}_{n-1}(s) \\
\mathbf{u}_{n}(s)
\end{array}\right)
$$

By using the Serret Frenet type formula again and again, we obtain the following lemma 2.3.

Lemma 2.3 For any $i \quad(0 \leq i \leq n-2)$, we have the following.

1. $\mathbf{u}_{i}(s) \cdot \frac{d^{j} \mathbf{u}_{n}}{d s^{j}}(s)=0 \quad(1 \leq j \leq n-i-1)$,
2. $\mathbf{u}_{i}(s) \cdot \frac{d^{n-i} \mathbf{u}_{n}}{d s^{n-i}}(s)=(-1)^{n-i} \prod_{j=0}^{n-i-1} \kappa_{n-j}(s)$.

\section{Explicit formula for the pedal curve relative to $\boldsymbol{P}$}

Let $\mathbf{r}$ be an $n$-dimensional spherical unit speed curve and let $P$ be any point in $S^{n}-\left\{ \pm \mathbf{u}_{n}(s) \mid s \in I\right\}$. By using the orthogonal frame $\left\{\mathbf{u}_{0}(s), \cdots, \mathbf{u}_{n}(s)\right\}$, we may decompose $P$ as

$$
P=\sum_{i=0}^{n}\left(P \cdot \mathbf{u}_{i}(s)\right) \mathbf{u}_{i}(s)
$$

\section{Lemma 3.1}

$$
\operatorname{ped}_{\mathbf{r}, P}(s)=\frac{1}{\sqrt{1-\left(P \cdot \mathbf{u}_{n}(s)\right)^{2}}}\left(P-\left(P \cdot \mathbf{u}_{n}(s)\right) \mathbf{u}_{n}(s)\right)
$$

Proof of lemma 3.1 For any $s \in I$, by subtracting $\left(P \cdot \mathbf{u}_{n}(s)\right) \mathbf{u}_{n}(s)$ from $P$ we obtain the vector $P-\left(P \cdot \mathbf{u}_{n}(s)\right) \mathbf{u}_{n}(s)$ in $\mathbf{R}^{n+1}$ which is positive scalar multiple of $\operatorname{ped}_{\mathbf{r}, P}(s)$. Normalizing this vector gives the right hand side of the formula in lemma 3.1, which must be the vector $\operatorname{ped}_{\mathbf{r}, P}(s)$.

By this formula, we have the following.

\section{Lemma 3.2}

$$
\operatorname{ped}_{\mathbf{r}, P}^{\prime}(s)=0 \Longleftrightarrow \kappa_{n}(s)=0 \text { or } P \in S_{\mathbf{u}_{n-2}(s)}^{n-2} .
$$


Proof of lemma 3.2 By differentiating $\operatorname{ped}_{\mathbf{r}, P}$ and using lemmata 2.2 and 3.1, we have the following.

$$
\begin{aligned}
& \operatorname{ped}_{\mathbf{r}, P}^{\prime}(s) \\
& =-\kappa_{n}(s) \frac{\left(P \cdot \mathbf{u}_{n}(s)\right)\left(P \cdot \mathbf{u}_{n-1}(s)\right)}{\left(1-\left(P \cdot \mathbf{u}_{n}(s)\right)^{2}\right)^{\frac{3}{2}}} \sum_{i=0}^{n-1}\left(P \cdot \mathbf{u}_{i}(s)\right) \mathbf{u}_{i}(s) \\
& \quad+\kappa_{n}(s) \frac{1}{\left(1-\left(P \cdot \mathbf{u}_{n}(s)\right)^{2}\right)^{\frac{1}{2}}}\left(\left(P \cdot \mathbf{u}_{\mathbf{n}}(\mathbf{s})\right) \mathbf{u}_{n-1}(s)+\left(P \cdot \mathbf{u}_{n-1}(s)\right) \mathbf{u}_{n}(s)\right) .
\end{aligned}
$$

Since $\left\{\mathbf{u}_{0}(s), \cdots, \mathbf{u}_{n}(s)\right\}$ is an orthogonal frame, we see that $\operatorname{ped}_{\mathbf{r}, P}^{\prime}(s)=0$ if and only if $\kappa_{n}(s)=0$ or $P \in S_{\mathbf{u}_{n-2}(s)}^{n-2}$.

Let $P$ be a point of $S^{n}-\left\{ \pm \mathbf{u}_{n}(s) \mid s \in I\right\}$. We consider the following $C^{\infty}$ map $\Psi_{P}: S^{n}-\{ \pm P\} \rightarrow S^{n}:$

$$
\Psi_{P}(\mathbf{x})=\frac{1}{\sqrt{1-(P \cdot \mathbf{x})^{2}}}(P-(P \cdot \mathbf{x}) \mathbf{x}) .
$$

We see that the image $\Psi_{P}\left(S^{n}-\{ \pm P\}\right)$ is inside the open hemisphere centered at $P$. Let this open hemisphere, the set $\pi\left(S^{n}-\{ \pm P\}\right)$ be denoted by $X_{P}, B_{P}$ respectively, where $\pi: S^{n} \rightarrow P^{n}(\mathbf{R})$ is the canonical projection. Note that $X_{P}$ is $C^{\infty}$ diffeomorphic to the $n$-dimensional open ball $\left\{\left(x_{1}, \cdots x_{n}\right) \mid \sum_{i=1}^{n} x_{i}^{2}<1\right\}$.

Since $\Psi_{P}(\mathbf{x})=\Psi_{P}(-\mathbf{x}), \Psi_{P}$ induces the map $\widetilde{\Psi}_{P}: B_{P} \rightarrow X_{P}$. Then, lemma 3.1 shows that $\operatorname{ped}_{\mathbf{r}, P}$ is factored into three maps in the following way.

$$
\operatorname{ped}_{\mathbf{r}, P}(s)=\widetilde{\Psi}_{P} \circ \pi \circ \mathbf{u}_{n}(s) .
$$

\section{Map of blow up type}

Let $p: B \rightarrow \mathbf{R}^{n}$ be the blow up of $\mathbf{R}^{n}$ centered at the origin.

Lemma 4.1 Let $P$ be a point of $S^{n}-\left\{ \pm \mathbf{u}_{n}(s)\right\}$. Then, there exist $C^{\infty}$ diffeomorphisms $h_{s}: B_{P} \rightarrow B$ and $h_{t}: X_{P} \rightarrow \mathbf{R}^{n}$ such that the equality $h_{t} \circ \widetilde{\Psi}_{P} \equiv p \circ h_{s}$ is satisfied.

By lemma 4.1 , it is reasonable to call $\widetilde{\Psi}_{P}$ a map of blow up type.

Proof of lemma 4.1 By a suitable rotation of $S^{n}$ if necessary, we may assume that $P=(0, \cdots, 1)$. For any $i \quad(1 \leq i \leq n)$ and any $\left(x_{1}, \cdots, x_{n+1}\right) \in S^{n}-\{ \pm P\}$ with $x_{i} \neq 0$ we put

$$
\varphi_{P, i}\left(\pi\left(x_{1}, \cdots, x_{n+1}\right)\right)=\left(\frac{x_{1}}{x_{i}}, \cdots, \frac{x_{i-1}}{x_{i}},-\tan (\lambda) x_{i}, \frac{x_{i+1}}{x_{i}}, \cdots, \frac{x_{n}}{x_{i}}\right),
$$

where $\lambda=\sin ^{-1}\left(x_{n+1}\right) \quad\left(-\frac{\pi}{2}<\lambda<\frac{\pi}{2}\right)$. Then, we see easily that for any $i, j \quad(1 \leq$ $i, j \leq n)$ the following equality holds

$$
\varphi_{P, j} \circ \varphi_{P, i}^{-1} \equiv \varphi_{j} \circ \varphi_{i}^{-1}
$$

where $\left\{\left(U_{1}, \varphi_{1}\right) \cdots,\left(U_{n}, \varphi_{n}\right)\right\}$ is the standard atlas for the blowing up $p: B \rightarrow \mathbf{R}^{n}$. Thus, the set

$$
\left\{\left(U_{P, 1}, \varphi_{P, 1}\right), \cdots,\left(U_{P, n}, \varphi_{P, n}\right)\right\}
$$


can be an atlas for $\pi\left(S^{n}-\{ \pm P\}\right)$, where $U_{P, i}=\left\{\pi\left(x_{1}, \cdots, x_{n+1}\right) \mid x_{i} \neq 0\right\}$.

Next, we express our map $\widetilde{\Psi}_{P}$ by using euclidean coordinates $\left(u_{1}, \cdots, u_{n}\right)$. Since we have assumed $P=(0, \cdots, 0,1)$, for $\mathbf{x}=\left(x_{1}, \cdots, x_{n}, \sin (\lambda)\right)$ we have

$$
\frac{1}{\sqrt{1-(P \cdot \mathbf{x})^{2}}}(P-(P \cdot \mathbf{x}) \mathbf{x})=\left(-\tan (\lambda) x_{1}, \cdots,-\tan (\lambda) x_{n}, \cos (\lambda)\right)
$$

and therefore for any $i \quad(1 \leq i \leq n)$ we have

$$
q \circ \widetilde{\Psi}_{P} \circ \varphi_{P, i}^{-1}\left(u_{1}, \cdots, u_{n}\right)=\left(u_{1} u_{i}, \cdots, u_{i-1} u_{i}, u_{i}, u_{i+1} u_{i}, \cdots, u_{n} u_{i}\right)
$$

where $q: \mathbf{R}^{n} \times \mathbf{R} \rightarrow \mathbf{R}^{n}$ is the canonical projection.

Since this expression is completely the same as that of the blow up by using the standard coordinate system $\left(U_{i}, \varphi_{i}\right) \quad(1 \leq i \leq n)$ and the restriction $\left.q\right|_{X_{P}}: X_{P} \rightarrow$ $q\left(X_{p}\right)$ is a $C^{\infty}$ diffeomorphism, we see that lemma 4.1 is proved for $\left.\widetilde{\Psi}_{P}\right|_{U_{P, i}}$ and $\left.p\right|_{U_{i}}$. Thus, in order to finish the proof of lemma 4.1 it suffices to show that for any $i, j \quad(1 \leq i, j \leq n)$ the equality

$$
\varphi_{i}^{-1} \circ \varphi_{P, i}\left(\pi\left(x_{1}, \cdots, x_{n+1}\right)\right)=\varphi_{j}^{-1} \circ \varphi_{P, j}\left(\pi\left(x_{1}, \cdots, x_{n+1}\right)\right)
$$

holds for $\pi\left(x_{1}, \cdots, x_{n+1}\right) \in U_{P, i} \cap U_{P, j}$. This holds since we have already checked that the patching relations for our $\left\{\left(U_{P, i}, \varphi_{P . i}\right)\right\}_{1 \leq i \leq n}$ are completely the same as for the standard atlas of $B$.

\section{Proof of theorem 1.1}

Since $\left\{S_{\left.\mathbf{u}_{n\left(s_{0}\right)}\right)}^{n}-S_{\mathbf{u}_{n-2}\left(s_{0}\right)}^{n-2}, S_{\mathbf{u}_{n-2}\left(s_{0}\right)}^{n-2}-S_{\mathbf{u}_{n-3}\left(s_{0}\right)}^{n-3}, \cdots, S_{\mathbf{u}_{0}\left(s_{0}\right)}^{0}-S_{\mathbf{u}_{-1}\left(s_{0}\right)}^{-1}\right\}$ gives a stratification of $S^{n}-\left\{ \pm \mathbf{u}_{n}\left(s_{0}\right)\right\}$, "if parts" of 1,2 of theorem 1.1 follows from "only if parts" of 1,2 of theorem 1.1. Thus, we show only "only if parts" in the following.

[Proof of "only if part" of 1] By lemma 3.2, $\operatorname{ped}_{\mathbf{r}, P}^{\prime}\left(s_{0}\right) \neq 0$ in this case. Thus, the map-germ $\operatorname{ped}_{\mathbf{r}, P}\left(s_{0}\right)$ is non-singular.

[Proof of "only if part" of 2] By a suitable rotation of $S^{n}$ if necessary, we may assume that $P=(0, \cdots, 0,1) \in \mathbf{R}^{n+1}$. Then, since $P \in S_{\mathbf{u}_{i}\left(s_{0}\right)}^{i}-S_{\mathbf{u}_{i-1}\left(s_{0}\right)}^{i-1}$, by a furthermore suitable rotation of $S^{n}$ if necessary we may assume that $\mathbf{u}_{n}\left(s_{0}\right)=(1,0, \cdots, 0)$, $\mathbf{u}_{n-1}\left(s_{0}\right)=(0,1,0, \cdots, 0), \cdots, \mathbf{u}_{i+1}\left(s_{0}\right)=(\underbrace{0, \cdots, 0}, 1, \underbrace{0, \cdots, 0})$; and $\mathbf{u}_{j}\left(s_{0}\right) \quad(0 \leq j \leq i)$ have the following form

$$
(n-i-1) \text { elements } \quad(i+1) \text { elements }
$$

$$
\mathbf{u}_{j}\left(s_{0}\right)=(\underbrace{0, \cdots, 0}_{(n-i) \text { elements }}, \underbrace{a_{(n-i) j}, \cdots, a_{n j}}_{(i+1) \text { elements }}),
$$

where $a_{n i} \neq 0$.

By lemma 2.3, we see that the following three hold for component function-germs $u_{0 n}, \cdots, u_{n n}$ of the map-germ $\mathbf{u}_{n}=\left(u_{0 n}, \cdots, u_{n n}\right):\left(I, s_{0}\right) \rightarrow S^{n}$.

1. For any $j \quad(0 \leq j \leq n-i-1)$, the lowest degree of non-zero terms of $u_{j n}$ is $j$.

2. For any $j \quad(n-i \leq j \leq n-1)$, the lowest degree of non-zero terms of $u_{j n}$ is more than or equal to $n-i$. 
3. The lowest degree of non-zero terms of $u_{n n}$ is $n-i$.

Therefore, by lemma 4.1 we see that the following two hold for component functiongerms $\psi_{1}, \cdots, \psi_{n}$ of the map-germ $\left(q \circ \widetilde{\Psi}_{P} \circ \varphi_{P, 1}^{-1}\right) \circ\left(\varphi_{P, 1} \circ \pi \circ \mathbf{u}_{n}\right):\left(I, s_{0}\right) \rightarrow \mathbf{R}^{n}$.

1. For any $j \quad(1 \leq j \leq n-i)$, the lowest degree of non-zero terms of $\psi_{j}$ is $n-i+j-1$, 2. For any $j \quad(n-i+1 \leq j \leq n)$, the lowest degree of non-zero terms of $\psi_{j}$ is $2 n-2 i$.

Let $\mathcal{E}_{1}$ be the set of all $C^{\infty}$ function germs with one variable $(\mathbf{R}, 0) \rightarrow \mathbf{R}, m_{1}$ be its subset consisting of all function-germs with zero constant terms. Then, $m_{1}^{n-i} \mathcal{E}_{1}$ is a finitely generated $\mathcal{E}_{1}$-module. We put $f(t)=t^{n-i}$ and apply the Malgrange preparation theorem (for instance, see [3], [6], [9]) to $m_{1}^{n-i} \mathcal{E}_{1}$ and $f$. Then we see that for any function-germ $g \in m_{1}^{n-i} \mathcal{E}_{1}$ there exists a certain $C^{\infty}$ function-germ $\psi$ such that

$$
g(t)=\psi\left(t^{n-i}, \cdots, t^{2 n-2 i-1}\right) .
$$

Thus, for our map-germ $\operatorname{ped}_{\mathbf{r}, P}:\left(I, s_{0}\right) \rightarrow\left(S^{n}, \operatorname{ped}_{\mathbf{r}, P}\left(s_{0}\right)\right)$ there exists a germ of $C^{\infty}$ diffeomorphism $h_{t}:\left(S^{n}, \operatorname{ped}_{\mathbf{r}, P}\left(s_{0}\right)\right) \rightarrow\left(\mathbf{R}^{n}, 0\right)$ such that

$$
h_{t} \circ \operatorname{ped}_{\mathbf{r}, P}(s)=(\underbrace{\left(s-s_{0}\right)^{n-i}, \cdots,\left(s-s_{0}\right)^{2 n-2 i-1}}_{(n-i) \text { elements }}, \underbrace{0, \cdots, 0}_{i \text { elements }}) \text {. }
$$

\section{References}

1. Arnol'd, V. I.: The geometry of spherical curves and the algebra of quaternions. Russian Math. Surveys. 50: 1-68(1995)

2. Arnol'd, V. I.: Simple singularities of curves, Proc. Steklov Inst. Math. 226: 20-28(1999)

3. Bröcker, TH., Lander, L. C.: Differentiable germs and catastrophes, London Mathematical Society Lecture Note Series 17, Cambridge University Press, Cambridge, 1975

4. Bruce, J. W., Gaffney, T., du Plessis, A. A.: On left equivalence of map germs. Bull. London Math. Soc. 16: 303-306(1984)

5. Bruce, J. W., Giblin, P. J.: Curves and Singularities (second edition), Cambridge University Press, Cambridge, 1992

6. Golubitsky, M., Guillemin, V.: Stable Mappings and Their Singularities, Graduate Texts in Mathematics no. 14, Springer-Verlag, Berlin, 1974

7. Mather, J. N.: Stability of $C^{\infty}$ mappings, III, Finitely determined map-germs, Publ. Math. I. H. E. S. 35, 127-156(1969)

8. Porteous, I. R.: Geometric Differentiation (second edition), Cambridge University Press, Cambridge, 2001

9. Wall, C. T. C.: Finite determinacy of smooth map-germs, Bull. London Math. Soc. 13, 481-539(1981) 